\title{
The influence of environmental and social factors on the parameters dynamics of the Gompertz function
}

\author{
A.V. Krementsova \\ Emanuel Institute of Biochemical Physics, RAS, Moscow, Russia \\ e-mail: akrementsova@mail.ras.ru
}

Key words: life-span, the Gompertz force of mortality, Strehler-Mildwan correlation

It is known that the life span distribution of humans and laboratory animals is well fitted by the Gompertz function. The analysis of demographic data makes it possible to evaluate both the contribution of natural environmental factors that have a time localized, for example, a crop failure or epidemic, and socially significant factors, for example wars or the achievement of medicine in the dynamics of the life span distribution. In the work, demographic data of the survival during the last 200 years of a several European countries were analyzed. Based on these data, the parameters of the Gompertz function were identified. The Strehler-Mildvan correlation dependences between these parameters were obtained. It is shown that, despite the fact that environmental factors can strongly influence to the dynamics of the Gompertz function parameters, they do not violate the Strehler-Mildvan correlation, while the influence of social factors leads to its violation. Currently there is an active discussion in the literature about the origin of the StrehlerMildvan correlation. Data are given that the correlation is a mathematical artifact, and it is not a reflection of real demographic patterns. To verify this statement, the parameters of the Gompertz function in the variation proposed by Gumbel were identified. It is shown that in this case also the correlation dependence between the parameters of the Gompertz function were observed. The results obtained make it possible to conclude that the dynamics of the life span distribution is not accidental, but it is a reflection of the internal patterns of aging. 